\title{
Faire entrer le réel en collision avec le romanesque : l'art du montage dans Retour à Kotelnitch et Un Roman russe d'Emmanuel Carrère
}

La production d'Emmanuel Carrère, qui a connu une nette inflexion du romanesque vers le biographique et l'autobiographique, est marquée par des fils conducteurs qui renseignent sur sa conception de l'individu et de la littérature. Ses textes portent la trace d'une fascination pour l'horreur et la folie, se manifestant par un passage ambigu entre le réel vécu, et l'imaginaire proche de la fiction pure. Carrère a d'abord créé des personnages adeptes de fabulation ou en proie à des délires et obsessions (comme dans La Moustache, 1986 ou La Classe de neige, 1995) pour ensuite s'intéresser à un fait divers : celui qui a éclaté en France en 1993 sous le nom de "l'affaire Jean-Claude Romand", quand ce dernier a choisi de tuer sa famille et tenté de mettre fin à ses jours plutôt que révéler les mensonges sur lesquels il avait construit toute sa vie d'adulte. ${ }^{1}$ L'Adversaire (2000), enquête à la première personne sur cette affaire, a marqué un tournant dans la production littéraire de Carrère. Depuis, il n'a produit que des œuvres de "non-fiction", appellation dont il constitue un farouche défenseur en opposition à la notion plus répandue d'autofiction, en soulignant le fait que la part de fiction dans ses textes autobiographiques est nulle. ${ }^{2}$ Attrait pour le vécu et le réel d'une part, fascination pour le romanesque d'une autre : les textes de Carrère, qu'ils soient de fiction ou ouvertement auto/biographiques, ont tendance à dresser le portait d'individus ayant vécu leur vie comme des personnages de roman - c'est le cas de Limonov (2011). Mais c'est probablement dans le diptyque film/texte que constituent Retour à Kotelnitch (2003) et Un Roman russe (2007) que cette empreinte du romanesque sur la production autobiographique est la plus visible. Publié en 2007, Un Roman russe relate comment, au début des années 2000, Carrère a accepté un projet de documentaire qui l'emmènerait à Kotelnitch, en Russie, sur les pas du dernier prisonnier de guerre, Andras Toma, qui venait d'être retrouvé et allait être rapatrié vers son village natal en Hongrie. À cette époque, Carrère, qui se sentait luimême prisonnier du projet qui l'avait accaparé pendant plusieurs années, a pensé qu'en s'immergeant dans un reportage, il pourrait échapper à ce couloir oppressant de secrets que constitue L'Adversaire :

Aujourd'hui je n'en veux plus. Je ne supporte plus d'être prisonnier de ce scénario morne et immuable, quel que soit le point de départ de me retrouver à tisser une histoire de folie, de gel, d'enfermement, à dessiner le plan du piège qui doit me broyer. Il y a quelques mois j'ai publié un livre, L'Adversaire, qui m'a tenu prisonnier sept ans et dont je sors exsangue. J'ai pensé : maintenant, c'est fini, je passe à autre chose. Je vais vers le dehors, vers les autres, vers la vie. Pour cela, ce qui serait bien, ce serait de refaire des reportages.

Je l'ai dit autour de moi et on n'a pas tardé à m'en proposer un. Pas n'importe lequel : l'histoire d'un malheureux Hongrois qui, fait prisonnier à la fin de la seconde guerre mondiale, a passé plus de cinquante ans enfermé dans un hôpital psychiatrique au fin fond de la Russie. On s'est tous dit que c'était un sujet pour toi, répétait avec enthousiasme mon ami journaliste, et bien sûr cela m'a exaspéré. Qu'on pense à moi à chaque fois qu'il est question

\footnotetext{
${ }^{1}$ Alors que sa famille croyait qu'il était médecin, Romand était sans emploi, endetté, et s'était fait piéger par un engrenage infernal de mensonges.

${ }^{2}$ Voir l'article de Nathalie Crom Télérama, "Et le réel, comment ça s'écrit ?" qui accorde une large section à Carrère (<http://www.telerama.fr/livre/l-invention-du-roman-verite,43020.php >). Carrère développe cette réflexion dans un entretien de mars 2009 pour Les Inrocks avec Nelly Kaprièlian (<http://www.lesinrocks.com/2009/03/08/actualite/entretien-avec-emmanuel-carrere-la-vie-des-autres$1143654 />)$.
} 
d'un type emmuré toute sa vie dans un asile de fous, c'est précisément ce dont je ne veux plus. Je ne veux plus être celui que cette histoire intéresse. N'empêche qu'évidemment, elle m'intéresse. Et puis cela se passe en Russie, qui n'est pas le pays de ma mère puisqu'elle n'y est pas née, mais le pays où on parle la langue de ma mère, une langue que j'ai un peu parlée enfant et ensuite complètement oubliée. ${ }^{3}$

On le voit, Un Roman russe porte la trace indélébile du projet qui a occupé Carrère pendant les années précédentes, et c'est cette trace - les mensonges, la folie et les fabulations, et leurs effets - qui va en constituer le fil conducteur et qui a également détourné le "projet Kotelnitch" de son but original. Comment alors, dans le déroulement de ce projet et sa construction, le romanesque prend-il le pas sur le réel, et pourquoi ?

Un rappel des étapes successives ayant mené au film et au roman sera nécessaire avant de se questionner sur les choix formels effectués par Carrère pour évoquer la part de fiction qui habite les trajectoires personnelles, y compris la sienne. Je me concentrerai sur les occurrences de juxtaposition et collision entre réel et romanesque que le romancier-cinéaste convoque pour chercher à donner du sens à ces voyages en Russie. Le film comme le texte sont en effet construits par juxtaposition de séquences et de couches temporelles et dialoguent dans un complexe jeu d'échos, qui inclut une prolifération d'autres images, de souvenirs, de personnes-personnages. Mon analyse cherchera à démontrer comment, par une esthétique du montage fidèle au sens que lui a donné Eisenstein, Carrère produit un diptyque où la fictionnalisation s'offre comme réponse possible à l'inouï du réel.

\section{L'apport du romanesque et du montage dans le(s) projet(s) Kotelnitch}

En octobre 2000, Carrère et son équipe partent pour Kotelnitch, une petite ville située à $800 \mathrm{~km}$ à l'est de Moscou, pour filmer le "retour à la vie" d'Andras Toma, fait prisonnier de guerre en 1944, qui a passé presque toute sa vie adulte dans l'hôpital psychiatrique de Kotelnitch, muré dans le silence et l'isolation (il ne parle que le hongrois et n'a jamais essayé d'apprendre le russe), pour être "trouvé" par hasard quelque cinquante ans plus tard. Là, ils filment l'hôpital, rencontrent le personnel médical, ont accès au dossier de Toma - mais aucune de ces pistes ne s'avère être à la hauteur de leurs attentes, et le projet ne semble pas aboutir. La veille de leur départ, dans le bar local, l'équipe fait la rencontre d'autres habitants, dont Ania, une jeune femme qui se distingue des autres jeunes de Kotelnitch: elle parle français ; son fiancé Sacha, dont on comprendra qu'il est à la tête des services secrets du FSB local (qui a succédé au KGB), refuse d'être filmé. Pour Ania, le mystère qui entoure Andras Toma est tissé de mensonges, et ressemble à une histoire d'espionnage. La rencontre fortuite avec l'équipe s'arrête là - c'est la veille du départ, et l'équipe a tout juste le temps de filmer Ania qui leur entonne une chanson accompagnée de sa guitare. Ces quelques heures suffisent toutefois pour qu'Ania et Sacha soient d'emblée perçus comme des personnages romanesques, qui embellissent le triste de quotidien de Kotelnitch. Le lendemain, dans le train sur le chemin du retour, Carrère et son équipe, en visionnant les images floues tournées la veille au bar, parlent de retourner à Kotelnitch pour faire un autre film : celui de la ville et ses habitants, dans cette Russie oubliée du début du vingt-et-unième siècle. Ils en parlent, vont prendre de la Vodka pour marquer ce projet, et oublient.

Le film Retour à Kotelnitch (2003) ne raconte pas ou presque pas l'histoire d'Andras Toma - ce documentaire, qui avait été commandé pour Envoyé Spécial et Télérama, sera

\footnotetext{
${ }^{3}$ E. Carrère, Un Roman russe (Paris : Gallimard, Folio, 2009 [P.O.L, 2007]), pp. 18-19, abrévié RR par la suite.
} 
diffusé sur France 2 en 2001 sous le titre "Le soldat perdu"4, et deviendra finalement le bonus du DVD de Retour à Kotelnitch. Cela ne raconte pas non plus le deuxième voyage à Kotelnitch, dessiné sur des bases incertaines mais qui se produit quand même, au printemps 2002, une fois que l'équipe a obtenu du financement. Lors de ce deuxième voyage, ils apprennent à mieux connaître la ville et ses habitants, dont un groupe de lycéens ; ils revoient Ania, devenue mère d'un petit garçon, et Carrère exprime sa déception en réalisant que celle qu'il s'était figurée comme personnage romanesque ait un quotidien si banal. L'équipe retourne à Paris et débute le montage du film, sans grand enthousiasme. À ce moment, en août 2002, Carrère apprend qu'Ania et son fils ont été sauvagement assassinés, à coups de hache, dans un contexte qui ne sera jamais élucidé (les théories possibles évoquent l'acte d'un fou ou des motifs politiques). C'est là que commence véritablement le projet du film qui deviendra Retour à Kotelnitch: s'y rendre une troisième fois pour rendre hommage à Ania, fin 2002, quarante jours après sa mort, comme le veut la tradition.

Le film est donc rendu possible par la manière dont le réel, dans toute sa brutalité, s'est immiscé dans un scénario qui ne semblait pas arriver à la hauteur des attentes de Carrère: "Ce qui est horrible aussi, c'est que la mort d'Ania et de son fils rend le film possible. Il raconte quelque chose, désormais. Nous allons retourner à Kotelnitch ..." (RR, p. 349). Le réel doit s'entendre dans le sens que lui donne Philippe Forest : non ce qui a lieu, mais ce qui déstabilise notre perception du monde et de nous-même : le réel est "ce reste dont la 'réalité' ne veut pas, où il n'a pas sa place." 5 Cet événement brutal, impossible à comprendre, semble concrétiser les angoisses exprimées dans les livres précédents de Carrère : l'emprisonnement mental, la peur de devenir fou, les morts brutales. Grâce à la nouvelle trame narrative qui s'est dessinée sous ses yeux, Carrère change alors l'orientation du projet. Mais au lieu de présenter le nouveau projet comme lissé, final, le film comme le livre choisissent de montrer ces imprévus, déceptions et échecs, et d'en démont(r)er les rouages. De manière significative, c'est au moment du montage de ce qui aurait dû devenir Retour que le film se fait et se défait simultanément: le montage, moment clé de la production d'un film, prend alors le sens bien plus large qu'Eisenstein lui a donné, à savoir la manière dont images et représentations acquièrent une signification globale, et pas seulement un travail de sélection et d'assemblage de morceaux de pellicule. Ici, la stupeur et le manque de significations qui se dessinent dans le film appellent une suite au projet, afin de lui donner sens. Cette suite, ce sera Un Roman russe.

Publié quelques années après la sortie de Retour à Kotelnitch, Un Roman russe est construit sur les fondations posées par le film et sur le trou à combler qui se dessine à la fin de Retour $^{6}$ : malgré son titre, Un Roman russe est un récit autobiographique, composé d'éléments vérifiables, une réflexion sur la recherche de l'identité et la construction de soi. L'auteur-narrateur prend comme point de départ ces voyages en Russie afin de dérouler les différents niveaux d'une histoire personnelle et familiale qui a également ses sources en Russie, dans le but de démêler ses relations avec la langue russe. La mère de Carrère, l'éminente historienne spécialiste de la Russie Hélène Carrère d'Encausse, est fille d'émigrés russes. Cette langue entendue lorsqu'il était enfant était celle de son grand-père paternel qui,

\footnotetext{
${ }^{4}$ Avec comme coup de publicité sensationnaliste la Une du magazine Télérama du 24 au 30 mars 2000, et son gros titre "L'incroyable histoire du dernier prisonnier de la seconde guerre". Cet article-reportage est reproduit dans E. Carrère, Il est avantageux d'avoir où aller (Paris : P.O.L., 2016) sous le titre "Le Hongrois perdu", pp. 151-173.

${ }^{5} \mathrm{Ph}$. Forest, Le roman, le réel. Un roman est-il encore possible? (Nantes : Plein Feux, 1999), p. 41. Plus loin, il écrit : "[...] le 'réel' est justement ce que la représentation, le langage, la fiction n'approchent que pour y découvrir le lieu d'un défaut, d'un manque, d'un déchirement qui les suscite mais dont ils ne peuvent rendre compte." (p. 48).

${ }^{6}$ Comme le note Thierry Durand dans “Emmanuel Carrère : l'étranger en soi”, The French Review $83: 3$ (Feb. 2010), pp. 574-588, p. 576.
} 
comme Andras Toma, a disparu à la fin de la deuxième guerre mondiale, très vraisemblablement car il avait collaboré sur le plan professionnel et intellectuel sous l'Occupation. Contrairement à Toma, le grand-père de Carrère n'a jamais été retrouvé, et ne le sera probablement jamais. Ėcrire ce livre, c'est donc "donner à ce fantôme une sépulture (RR, p. 69), redoublant ainsi le film Retour à Kotelnitch qui tente de faire le deuil d'Ania. L'acte créatif acquiert donc une place essentielle dans ce processus de deuil, ${ }^{7}$ et le chassécroisé entre le projet Kotelnitch initial et le livre permet de mettre à jour des échos saisissants entre la situation de Toma et celle de la famille de Carrère. La famille d'Andras Toma a dû faire son deuil sans preuve de son décès, après avoir espéré son retour pendant des années, a accepté le verdict des autorités qui l'ont déclaré mort, puis a dû faire face au choc de le voir retourner dans le monde des vivants. En parallèle, Carrère explique que sa famille s'est prise elle aussi à 'imaginer l'inimaginable : qu'il vivait quelque part, qu'il était prisonnier peutêtre, qu'un jour il reviendrait. Aujourd'hui encore, je le sais parce qu'elle [=ma mère] me l'a dit, il lui arrive de rêver son retour." (RR, p. 71) Le diptyque agit donc comme manière de léguer à la famille de Carrère la sépulture devenue vide quand Toma a été retrouvé, en tentant de donner une matière tangible à la stupeur et l'incompréhensible que constitue la disparition d'un être proche.

D'emblée, ce projet présente un intérêt dans sa construction et ses ressorts : ni purement biographique ni autobiographique, à la fois ancré dans le présent et le passé, en France et en Russie, prenant appui sur l'histoire des autres pour parvenir à la sienne, le film puis le livre opèrent des mouvements spatio-temporels qui contribuent à leur rythme et à leurs différents niveaux d'interprétation. Pour le lecteur-spectateur, la réception du projet Kotelnitch nécessite un travail de juxtaposition et de brassage des différentes séquences texte et film, mots, images et chansons, époques et temps - afin de lui donner une cohérence. Il s'agit d'une part d'un travail traditionnel de montage, de mise en disposition des séquences, comme l'a expliqué Carrère au sujet de Un Roman russe: "Oui, mais encore une fois ma ruse était de me dire 'je n'écris pas ce livre, tout est déjà écrit, je n'ai plus qu'à monter, couper, faire entrer en résonance une chose avec une autre." ${ }^{8}$ Par ailleurs, dans Retour à Kotelnitch, Carrère explique quel aurait dû être le film qui n'a finalement pas été fait : au final, c'est cent heures d'images qui ont été tournées et qui n'ont pas été utilisées. On nous en montre quelques-unes sous forme de patchwork, dont celles du banquet avec les lycéennes, ouvertures possibles vers des films qui n'auront jamais lieu sauf dans la tête du spectateur. En plus de ces images qui ne verront jamais le jour, le film inclut une séquence sur la construction du film qui est elle-même un rouage du processus de construction : on y voit le travail de pré-montage du film, en l'occurrence un mur rempli de post-its avec le titre des séquences choisies et leur accompagnement en musique. Dans la constitution du film et son esthétisme, se manifeste donc un projet non scripté, heurté, ouvert à l'accidentel et aux collisions - terme emblématique de la théorie filmique d'Eisenstein. Lors du montage traditionnel, Carrère opère donc un travail de condensation et de mise en cohérence du film, tout en gardant ses fractures, comme pour mettre l'attention sur les moments où réel et romanesque entrent en collision, se touchant pour ensuite se disloquer.

Toutefois, si l'on suit le raisonnement d'Eisenstein, le montage n'est pas purement la sélection et l'assemblage d'images ou de séquences, mais doit s'entendre comme processus holistique liant différents fragments et leur donnant de la signification, par une démarche à la fois intellectuelle et émotionnelle. ${ }^{9}$ Dans ce sens, le rôle du destinataire est clé dans tout

\footnotetext{
${ }^{7}$ Il s'agit là d'un trait de nombreuses autofictions récentes, dont les textes de Doubrovsky et de Forest.

${ }^{8}$ Entretien avec Angie David, in Emmanuel Carrère, Editions Léo Scheer, Coll. Ecrivains d'aujourd'hui, 2007, p. 37.

9 On consultera en particulier son article de 1938, traduit en anglais par "Word-image" et disponible dans S. Eisenstein, The Film Sense, ed. et trad. Jay Leydan (London : Faber \& Faber, 1986 [1943]). Eisenstein y expose
} 
processus de montage, puisque c'est le lecteur-spectateur qui a la capacité d'unir des séquences à la fois temporelles et spatiales. Dans le cas de Retour à Kotelnitch et Un Roman russe, le travail à apporter face au fragmentaire et au non-dit démarche reproduit les errements et les difficultés de Carrère face à un projet semé de difficultés et d'inattendu - et in fine, reproduit sa propre position de spectateur, comme nous le verrons. Par ailleurs, faire sens de ces textes, c'est naviguer parmi les multiples oscillations entre factuel et fictionnel qui les sous-tendent.

\section{Un "roman vrai"}

Un Roman russe est un texte dont l'auteur a affirmé à plusieurs reprises qu'il ne s'agit pas d'un roman, et pourtant il en porte la mention dans son titre. En quoi ce romanesque consiste-t-il ? Bien qu'il milite pour l'appellation non-fiction et revendique la part de vérité dans ses textes les plus récents, Carrère propose dans son œuvre un rapport complexe entre vécu et romanesque. Son approche ne semble guère éloignée de la conception de l'autofiction selon Doubrovsky. Lorsque Carrère ne raconte que des "histoires vraies" en ne changeant pas les noms des personnages, y compris le sien, il utilise toutefois les ressources du roman: "Personnellement, j'ai l'impression de faire un travail de narration qui n'est pas du tout de la fiction, mais en utilisant exactement les mêmes procédés romanesques." 10 À propos de Un Roman russe, il a déclaré : "Remarquez, mon livre est construit de façon romanesque. Mais tout est vrai, à l'exception de quelques broutilles." "11 Sous ses airs de pirouette, cette remarque n'est pas sans rappeler l'approche doubrovskienne de l'autofiction : chez ce dernier, la forme est inventée et le contenu est vrai... à cent pour cent ou presque. ${ }^{12}$

Dans le diptyque film-livre, le rapport entre vrai et faux est compliqué par le fait que, bien que Carrère s'y affiche auteur d'un travail autobiographique, le mensonge, les énigmes et les secrets constituent une part importante du contenu des textes. Les éléments "vrais" sont, dans l'ensemble, dignes d'être vécus par des personnages de roman - ou de film, puisque "le cinéma est devenu synonyme de fiction", comme l'a remarqué Edgar Morin ${ }^{13}$ - et frappent par leur caractère inédit. La découverte d'Andras Toma, que tout le monde croyait mort, après cinquante ans passés en hôpital psychiatrique au fin fond de la Russie ; la disparition du grand-père de Carrère au sortir de la deuxième guerre mondiale, probablement victime de représailles ; 1'assassinat brutal d'Ania et son jeune enfant dans des circonstances obscures : ces trois trames semblent ne pas nécessiter d'apport fictif ou romanesque, se lisant déjà comme des histoires sensationnelles comme celles qui alimentent les pages fait divers des journaux. Le romanesque est également visible dans la manière même d'appréhender le réel, de l'anticiper, d'y coller ses propres attentes et espoirs, de telles sortes que ces derniers finissent par former un écran qui transforme le vécu. Lorsqu'il se rend à Kotelnitch la

en quoi la production d'images chargées de signification est un processus mêlant les intentions de l'auteur, celles de l'acteur et les conditions de réception par le spectateur.

${ }^{10}$ Voir Nicolas Marcadé et Chloé Rolland, "Le ‘je’ de la vérité”, Entretien avec Emmanuel Carrère, juillet 2009, <http://www.fichesducinema.com/spip/spip.php?article1442>.

${ }^{11}$ Cité dans Baptiste Liger, "Le petit-fils de l'empire éclaté", L'Express, mars 2007,

<http://www.lexpress.fr/culture/livre/un-roman-russe_812022.html>.

${ }^{12}$ Doubrovsky, qui se targue d'écrire sur des faits strictement réels, a en fait entièrement inventé une séquence centrale de Fils (un rêve) pour les besoins de la cause romanesque. Philippe Lejeune, en découvrant cela, s'est senti dupé, comme le rappelle Philippe Gasparini dans Autofiction, une aventure du langage (Paris : Seuil, 2008), pp. 78-79.

${ }^{13}$ E. Morin, Le cinéma ou l'homme imaginaire. Essai d'anthropologie sociologique (Paris : Minuit, Coll. Arguments, 2002 [1956]), p. 83. 
première fois avec son équipe pour faire un reportage sur Toma, Carrère semble s'imaginer que tout sera à la hauteur de cette trame romanesque, et qu'ils repartiront avec des images et anecdotes inoubliables. La réalité est tout autre : l'accueil qu'on leur réserve n'est pas des plus chaleureux, le dossier médical de Toma est factuel et laconique. Tous les espoirs sont alors placés dans un banquet organisé à l'attention des employés de l'hôpital qui se déroule justement à ce moment :

J'attends beaucoup de ce banquet, j'imagine une plongée haute en couleurs dans la Russie profonde, dont le clou, entre toasts enthousiastes et danses à perdre haleine, pourrait être la rencontre d'une vieille infirmière à la retraite, truculente babouchka qui nous raconterait l'arrivée du Hongrois en 1947 et nous laisserait entendre, l'œil pétillant de malice, qu'il avait beau ne rien dire, il avait plus d'un tour dans son sac, le grand coquin. (RR, pp. 40-41)

Or non seulement l'équipe de tournage n'est pas conviée, mais quand ils s'y immiscent quand même, profitant que le banquet se déroule dans leur hôtel, ils provoquent certes l'étonnement, mais ne créent aucun incident digne d'être relaté. L'imaginaire cinématographique du narrateur tente alors de pallier cette déception en donnant de la vie à la scène, en cherchant à décrire le film de ce qui aurait pu se passer :

Se déroule alors une scène de film burlesque : nous traversons la salle avec des petits sourires polis, des gestes bénins et rassurants qui veulent dire quelque chose comme : nous ne faisons que passer, faites comme si de rien n'était, ne vous dérangez pas. On nous suit des yeux avec effarement, et notre conduite à ce moment-là est tellement absurde qu'elle désarme toute agressivité. Dans un film, les héros détaleraient comme des lapins l'instant précis où, l'hypnose cessant de jouer, la horde se rue sur eux pour les écharper. (RR, pp. 48-49)

Cette séquence est emblématique des deux premiers séjours à Kotelnitch, qui font état de collisions entre des scénarios rêvés d'une part et le réel, dans ce qu'il a de plus ordinaire et tranquille, d'autre part. Les moments de tension qui intéressent Carrère sont ces points où, soudainement, réel et romanesque ne peuvent plus cohabiter parce qu'il devient impossibles de les distinguer, parce qu'une brèche s'ouvre dans l'existence. Le cinéma devient alors non seulement un outil pour capter le vécu, mais aussi une manière de dire le romanesque, comme si Carrère reprenait à son compte l'expression "se faire un film" en faisant de cinéma un synonyme de "inventé" ou "incongru". Le chassé-croisé entre réel et images va alors fonctionner par l'inclusion, dans le livre, de séquences à tonalité cinématographiques pour rendre le réel plus palpitant et dans le film, par des séquences on ne peut plus laconiques et banales où rien ne semble se produire. En effet, Un roman russe regorge de séquences narrant des projections imaginaires comme celles évoquées plus haut, qui seront déboutées par le réel. C'est par l'imagination que le lecteur va se les projeter, étape supplémentaire au montage déjà effectué par l'auteur. Dans le film, puisque les images ne peuvent pas montrer ce qui n'a pas lieu, c'est par des moments de pause, où Carrère se trouve seul devant la caméra, que se déploie un espace d'imagination et d'autoréflexion. Le cinéaste, qui se fait alors auteur-acteur, se met en scène en tant que spectateur de ces collisions et l'image cinématographique devient alors, pour citer Morin, "l'acte constitutif radicalement simultané du réel et de l'imaginaire". ${ }^{14}$ Ces moments de pause ouvrent sur un nouvel espace spatiotemporel qui n'est pas présent dans le film mais qui sera développé dans Un Roman russe : la quête des origines.

\footnotetext{
${ }^{14}$ Morin, p. x. En italiques dans le texte.
} 


\section{Roman des origines, origines du roman}

La part de romanesque de Un Roman russe est aussi à trouver dans l'histoire familiale qui se transforme véritablement en roman des origines. Carrère possède peu d'éléments vérifiables et sûrs au sujet de sa famille, à l'époque de la disparition de son grand-père. Il entreprend donc de retracer le parcours de son grand-père en assemblant archives et anecdotes à son sujet. Mais il est clair que cette tentative biographique accorde une grand part aux histoires familiales devenues mythiques, et qu'elle sélectionne les éléments les plus romanesques ou inédits : son grand-père a été chauffeur de taxi à Paris dans les années vingt, et la légende veut qu'il préférait refuser des courses plutôt que devoir abréger la lecture d'un ouvrage de philosophie ; il est perçu comme "un vrai intellectuel russe" $(R R, \mathrm{p} .91)$, et dans ses lettres se dessine un personnage digne de Dostoïevski $(R R$, p. 96); la grand-mère est comparée à un personnage d'un livre de Katherine Mansfield ( $R R$, p. 92); la mère aime raconter l'histoire improbable selon laquelle, grandissant en France dans un milieu d'émigrés russes, elle aurait cru être en Russie jusqu'à l'âge de quatre ans; et le narrateur, pensant à l'aspect indigent de son grand-père avec sa mère enfant, les imagine assis dans le métro, soumis aux regards des plus aisés. Cette scène qu'il compare à des "romans misérabilistes" ( $R R$, p. 106) n'a qu'un ancrage ténu dans la réalité, et le narrateur en est conscient : "J'imagine ces mots, et peut-être cette scène. Il me semble que pourtant ma mère m'a un jour raconté quelque chose de ce genre.” ( $R R$, p. 107) Mais au lieu de la considérer comme fabulation, il serait peut-être plus juste de percevoir cette scène comme bribe de mémoire collective familiale qui s'est créée et transmise, au fil des années - mémoire qui a intégré souffrances et humiliations, mais aussi une manière de vivre le quotidien au prisme du romanesque, peut-être pour l'embellir. Ou post-mémoire (postmemory), pour utiliser la notion théorisée par Marianne Hirsch, qui se réfère aux souvenirs dont hérite une génération qui suit celle qui a vécu un épisode traumatisant.

C'est dans le cadre de réflexions sur l'Holocauste que Hirsch développe cette notion, et il est évident que dans le cas de Carrère, la disparition de son grand-père après l'Occupation est l'élément déclencheur qui a teinté les souvenirs de sa famille et "l'inconscient de deux générations" $(R R$, p. 392) - il parle même de "malédiction de la famille." ( $R R$, p. 392) Pour Hirsch, l'imaginaire est un élément central du processus de postmémoire :

In my reading, postmemory is distinguished from memory by generational distance and from history by deep personal connection. Postmemory is a powerful and very particular form of memory precisely because its connection to its object or source is mediated not through recollection but through an imaginative investment and creation. [...] Postmemory characterizes the experience of those who grow up dominated by narratives that preceded their birth, whose own belated stories are evacuated by the stories of the previous generation shaped by traumatic events that can be neither understood nor recreated. ${ }^{15}$

La mémoire familiale de Carrère, toute teintée de fabulation et de romanesque qu'elle est, ne se lit pas moins comme authentique et révélatrice des dynamiques complexes qui structurent cette famille et sa transmission du souvenir. Le romanesque se trouve ainsi tissé dans ce mode de transmission intergénérationnelle, nous donnant à lire un véritable roman des origines. Marthe Robert a théorisé le roman des origines comme roman faisant la part belle aux mythes fondateurs, directement hérités de l'enfance, un roman qui défie les classements génériques: "Le mythe familial de l'enfance définit donc le roman dans ce qui le rend

\footnotetext{
${ }^{15}$ M. Hirsch, Family frames: photography, narrative and postmemory (Cambridge, MA : Harvard University Press, 2012 [1997]), p. 22.
} 
précisément indéfinissable : son absence de caractères génériques [...] et, surtout, le désir de faire vrai [...]." ${ }^{16}$ Dans Un Roman russe, on apprend que les parents de Carrère aiment raconter le parcours de la gouvernante, "qui était digne d'un roman d'aventures" $(R R$, p. 145) : née dans une famille de tzigane, ayant évolué dans des cabarets, elle aurait vécu avec un prince, émigré après son assassinat, et une fois à Paris, aurait sympathisé avec Coco Chanel pour finir gouvernante sur la recommandation des Tolstoï. Là non plus, rien de vérifiable : le lecteur doit juste accepter la parole de celui qui raconte. Et le jugement de l'auteur-narrateur, celui qui connaît parfaitement les méandres du mensonge et ses trouées dans le réel pour les avoir écrits dans L'Adversaire, est des plus bienveillants, suggérant que derrière "des incohérences qui n'étaient pas forcément des mensonges" ( $R R, \mathrm{p} .146)$, c'est au lecteur d'accepter sa position délicate : "on croit ou non ce genre de récits, mais on ne les contrôle pas." (RR, p. 146) Nous avons ici affaire à un pacte autobiographique d'un genre différent, dont la souplesse réside dans cet élément majeur : le fait que le lecteur ou l'auditeur sont avides d'histoires inédites, et que le romanesque entre certes en collision, mais pas en contradiction, avec le réel ou le vécu.

Le personnage d'Ania est à lui seul la synthèse de mouvements du quotidien au romanesque. Le film et le livre ne cachent pas le fait que les séjours à Kotelnitch relatent de nombreux non-événements, d'obstacles et d'échecs, eux-mêmes comme anticipés et rendus possibles par un non-scénario. C'est parce qu'ils ne peuvent participer au banquet de l'hôpital que Carrère et son équipe vont se rendre au bar local et y rencontrer Ania et Sacha. Charmé par ce couple, Carrère est déçu, lors du deuxième séjour, de réaliser que le côté romanesque d'Ania semble surtout se limiter à son aspect "gentille fille naïve, bovarysante, sentimentale" (RR, p. 237) :

Ania m'a un peu déçu, le jour de ces retrouvailles. J'attendais la Mata Hari de Kotelnitch, je me suis retrouvé devant une jeune mère qui me paraissait banale et à qui je ne savais plus très bien quoi dire. Pourtant, je garde de mon premier séjour, de notre nuit d'ivresse à la Troïka, la conviction qu'un mystère les entoure, Sacha et elle, en tout cas une aura romanesque. ( $R R, \mathrm{p}$. 227)

Après sa mort tragique, lorsque Carrère rencontre sa mère, Ania acquiert enfin la profondeur qu'il espérait trouver chez elle : à ce moment, on apprend qu'elle aimait fabuler, et qu'elle aussi, comme Carrère, aimait à projeter ses désirs sur un écran - dans le cas de Carrère, c'est la Russie (son histoire, sa langue, sa complexité) qui joue ce rôle d'écran, tandis que dans le cas d'Ania, c'est la France. Retour aux origines mythiques pour l'un (avec des trous à combler), ${ }^{17}$ manière de se créer d'autres origines, plus romanesques, pour l'autre : on apprend qu'au début de sa liaison avec Sacha, Ania lui a expliqué “qu'elle était à moitié française, par sa mère qui était morte en couches, et qu'elle possédait même une maison près de Paris, où elle se rendait fréquemment. Déjà impressionné par sa connaissance du français, Sacha l'a été encore plus par ces révélations." ( $R R, \mathrm{p}$. 365) Plus loin, Sacha explique qu'Ania lui a raconté qu'elle aurait été une espionne française, histoire à laquelle il semblait vouloir croire. Et là encore, le narrateur suspend son jugement quant à la véracité de ces contes - ou plutôt, il semble se ranger du côté de ceux qui préfèrent teinter le réel d'une couche de fiction :

Je n'avais pas eu tort, décidément, de les trouver romanesques dès notre première rencontre, de l'appeler en plaisantant la Mata Hari de Kotelnitch. Ensemble, ils s'étaient raconté un roman dans lequel ils vivaient, elle étant le moteur et lui la suivant dans ses fabulations parce qu'au fond, comme je l'avais tôt deviné, cela lui plaisait. ( $R R$, p. 380)

\footnotetext{
${ }^{16}$ M. Robert, Roman des origines et origines du roman (Paris : Gallimard, Coll. Tel, 1981 [1972]), p. 64.

${ }^{17}$ Hirsch explique se sentir proche de la notion de "mémoire trouée" de Henri Raczymow, ces trous qui définissent "the indirect and fragmentary nature of second-generation memory." Hirsch, p. 23.
} 
"Un roman dans lequel ils vivaient" : sans le vouloir, en allant à Kotelnitch, d'accidents de parcours en déceptions, le narrateur a retrouvé ce qu'il voulait justement fuir après l'écriture de L'Adversaire : des personnages en proie à des fabulations qui mènent, directement ou non, à une issue tragique, où le réel dépasse le romanesque.

\section{Performance de l'imaginaire}

Ce qu'Un Roman russe dit que Retour à Kotelnitch ne disait pas, c'est aussi la face privée de ce qui se jouait dans la vie de Carrère au moment de ce projet, de ces voyages et obsessions : sa relation tumultueuse avec une jeune femme prénommée Sophie, relation dont on peut se demander pourquoi elle occupe une place si importante dans le récit, en dehors du fait qu'elle contient les ingrédients idéaux - passion, indifférence, jalousie - pour une histoire d'amour romanesque. Au cœur de Un Roman russe, Carrère a reproduit une nouvelle qu'il avait écrite pour Le Monde et qui fut publiée le 20 juillet 2002 sous le titre "L'usage du Monde"; nouvelle écrite en réponse à une demande du quotidien, dans le cadre de suppléments d'été sur le thème du voyage. Carrère s'est littéralement pris au jeu, en faisant de ce texte un enjeu essentiel de sa relation avec Sophie : la nouvelle, écrite à la première personne, s'adresse à un "tu" qui prend un train Paris-La Rochelle, lui donne une suite de consignes érotiques qui devrait culminer dans une atmosphère de sensualité extrême dans le wagon-bar du train, où se retrouveraient tous ceux et celles qui lisent la nouvelle, et dans les toilettes, où se rendraient tous ceux pris d'un désir irrépressible : “[...] voilà, ce jeudi 23 mai j'ai décidé que le samedi 20 juillet, dans le train de $14 \mathrm{~h} 45$ pour La Rochelle, la femme que j'aime se branlera en suivant mes instructions et jouira entre Niort et Surgères [...]." (RR, p. 179) Dans la nouvelle, à aucun moment le "tu" n'est identifié comme étant Sophie, mais Carrère explique dans Un Roman russe qu'il avait réservé un billet pour que Sophie prenne effectivement ce train, et que tout se déroule comme prévu. Stratégie risquée que cette littérature performative, quand une projection osée doit quitter le domaine de la fabulation pour entrer en contact avec la réalité, pour se réaliser. ${ }^{18}$ Le contenu de la nouvelle ne peut s'accomplir que si toutes les consignes sont respectées à la lettre, ce qui est évidemment peu probable. Pour les journalistes du Monde qui ont eu la primeur de la lire avant la date de publication, ce fut aussi l'occasion de savoir si le contexte de cette nouvelle est inventé : s'adresse-t-elle à une femme réelle, ou est-ce une pure invention ? Carrère, qui a décidé de prendre lui aussi ce train pour observer l'impact de sa nouvelle sur les voyageurs, s'est trouvé pris à son propre jeu par une équipe de journalistes du Monde, envoyés dans le train et forcés de constater que cette jeune femme n'est pas au rendez-vous, alors que Carrère, lui, avait pris place dans le train.

Sophie, en effet, ne prendra pas le train, et ce rendez-vous raté entre l'imaginaire et le réel, ainsi que la faillite du dispositif qui l'accompagne, signalent la rupture à venir du couple. La réalité se situe on ne peut plus loin des effets anticipés par Carrère, sûr du succès de sa nouvelle, au point de projeter sa félicité à venir avec Sophie en croyant s'être débarrassé des anciens démons: "Fini les histoires de folie, de perte, de mensonge, enfin je suis passé à autre chose, je dis à une femme que je l'aime, c'est une déclaration d'amour." ( $R R$, p. 253) Comme le souligne Fabien Gris, Un Roman russe offre à réfléchir sur le désir de contrôle auctorial et la part d'éléments incontrôlables : bien que la nouvelle "L'usage du Monde" soit un "dispositif performatif extrêmement calculé", elle va finalement confronter Carrère à sa propre "impuissance quant à la maîtrise [...] du réel." ${ }^{19}$ Cette nouvelle

\footnotetext{
18 Cette littérature performative, ce “dispositif” (RR, p. 182) savamment orchestré, nous font penser inévitablement aux expérimentations performatives d'une autre Sophie : Sophie Calle.

${ }^{19}$ F. Gris, "Emmanuel au carré", Roman 20:50, 57 (juin 2014), pp. 35-46, p. 42.
} 
"performative" n'a peut-être pas eu les effets escomptés, mais elle a eu des effets sur le narrateur et sa relation amoureuse. Surtout, son inclusion au cœur du livre - qui peut surprendre dans le cadre général d'une quête des origines - nous rappelle que cette quête est d'abord une plongée dans l'imaginaire : l'imaginaire personnel de Carrère, l'imaginaire familial, et l'imaginaire collectif au sujet de la Russie. Par conséquent, ce qui relève du fantasme, du rêve, et surtout la collision entre les projections fantasmées et le vécu, doivent être inclus dans cette quête car ces éléments sont, eux aussi, tangibles. On touche au noyau de ce qui relie, inextricablement, fiction et réel dans ces projets artistiques : à savoir, la fiction comme mode d'existence, et un réel qui n'est pas synonyme de réalisme mais d'inouï. ${ }^{20}$

\section{Conclusion}

Alors qu'il "boucle" Un Roman russe, le narrateur écrit : "Mais je n'écris pas un roman [...]." $(R R$, p. 391) Il s'agit bien, en effet, d'entreprise autobiographique, de tentative pour mieux se comprendre ainsi que ses origines en allant au plus profond de soi, en plongeant dans des rêves, bribes de souvenirs d'enfance, fantasmes, éléments de mythologie familiale. Mais la fiction est le vecteur de ces retours aux origines pour des personnages qui traversent des histoires vraies qui ressemblent à des romans, qui aiment se raconter des histoires et rendre romanesque non nécessairement leur existence, mais surtout le récit de celle-ci. Comme l'a remarqué Dominique Rabaté, "Le réel ne répond plus que par l'extrême violence (cruauté de la rupture avec Sophie, brutalité du massacre à la hache d'Ania et de son fils) mais sans mettre un terme à l'activité fantasmatique de ses acteurs, réactivant les scénarios trop rodés des vies parallèles et secrètes." 21 Dans sa saga russe, quand le réel ne se déroule pas de manière romanesque ou inédite, quand les voyages sont sources de déceptions, Carrère choisit de l'écrire comme un roman, c'est-à-dire, dans son cas, avec le recours à l'univers filmique, où les projections imaginaires l'emportent souvent sur le vécu, au risque de s'entrechoquer avec le réel. Chez Carrère, ces deux trajectoires inverses (écrire l'inédit de manière factuelle et écrire le banal de manière romanesque) se rejoignent dans une zone de friction qui correspond aux potentiels de la production autofictionnelle - celle où le crédible est une notion sans cesse remise en question, où le lecteur comme le narrateur sont obligés de suspendre leurs doutes :22 “Je ne te dis pas que c'est vrai, je te dis juste qu'en Russie c'est un genre d'histoire qui arrive." (RR, p. 363), comme le déclare à Carrère son collègue Philippe. On se situe dans une lignée ouverte avec des pratiques qui ont contribué à renouveler l'écriture de soi, comme le note Gasparini quand il évoque les enjeux de la nouvelle autobiographie chez Robbe-Grillet: "Il ne s'agit plus pour lui de rénover, de réformer, de moderniser un genre noble mais de transformer une forme de 'reportage' en écriture littéraire, donc en écriture romanesque, car, dans sa poétique, il n'y avait de littéraire que romanesque." ${ }^{23}$ Et mettre en suspens ses doutes ou son incrédulité est peut-être la seule manière de se plonger dans des récits où le réel peut sembler moins plausible que la fiction. Chez Carrère, le romanesque se situe non seulement dans la composition des livres et films (montages et juxtapositions qui visent l'émotion, construction élaborée, scénarisation, phénomènes d'attentes et d'anticipation, prolepse et analepse), mais surtout dans la mise par écrit d'épisodes choisis d'une vie qui sont retranscrits (car comment savoir qu'ils ont vraiment été vécus de la sorte ?) au travers du prisme de l'invention. Et surtout le romanesque

\footnotetext{
${ }^{20}$ Philippe Forest évoque "l'appel inouï du réel" que la littérature cherche à saisir. In Le roman, le réel, p 21.

${ }^{21}$ D. Rabaté, "Passages à la limite. Roman et romanesque chez Emmanuel Carrère", in Chercher la limite, ed. Matteo Majorano (Bari : Edizioni B.A. Graphis, 2008), pp. 65-78, pp. 77-78.

${ }^{22}$ Il est dommage qu'il n'existe pas en français d'expression qui corresponde à l'anglais "suspension of disbelief", qui exprime cette nécessité de croire à l'invraisemblable.

${ }^{23}$ Gasparini, p. 144.
} 
chez Carrère est un romanesque où la récurrence du cinématographique et l'art du montage appuient la part d'imaginaire qui habite ses productions. 